\title{
Magneto-Optical Studies of Narrow Band-Gap Heterostructures with Type II Quantum Dots InSb in an InAs Matrix
}

\author{
M.S. Mukhin ${ }^{a, b, *}$, Ya.V. Terent'ev ${ }^{a}$, L.E. Golub ${ }^{a}$, M.O. Nestoklon $^{a}$, B.Ya. Meltser ${ }^{a}$, \\ A.N. Semenov ${ }^{a}$, V.A. Solov'Ev ${ }^{a}$, A.A. Sitnikova ${ }^{a}$, A.A. Toropov ${ }^{a}$ And S.V. Ivanov ${ }^{a}$ \\ ${ }^{a}$ Ioffe Physical-Technical Institute of Russian Academy of Sciences \\ 26 Polytekhnicheskaya, St Petersburg 194021, Russian Federation \\ ${ }^{b}$ St Petersburg Academic University - Nanotechnology Research Centre of Russian Academy of Sciences \\ 8/3 Khlopina, St Petersburg 194021, Russian Federation
}

\begin{abstract}
Magneto-optical properties of type II heterostructures with InSb/InAs quantum dots has been studied at external magnetic field applied in the Faraday geometry. The emission polarization degree can be changed in the range from $100 \% \sigma$-minus to $10 \% \sigma$-plus due to excitation intensity and temperature variation. The detailed calculation of the band structure within a tight-binding approximation is presented. The simulation of the experimental data reveals that the oscillator strength of the optical transitions involving electrons with the spin oriented along and opposite to the magnetic field vector differs by approximately 1.8 times in the heterostructures under study.
\end{abstract}

PACS: 75.75.-c, 71.70.Ej, 78.55.Cr, 78.67.Hc, 78.20.Ls, 75.40.Mg

\section{Introduction}

Spin is the only electron internal degree of freedom, and utilizing it in the new generation of semiconductor devices is the main goal of semiconductor spintronics. Today spintronics focuses mainly on diluted magnetic semiconductors (DMS) where ferromagnetism and giant Zeeman splitting can be obtained due to exchange interaction between free carriers and Mn ions. Most of the work has been focused on II-VI DMS such as CdMnTe or ZnMnSe and some others. Enhanced magnetic properties of these materials exist only at cryogenic temperatures due to paramagnetic behavior of the magnetic ions. A lot of recent investigations have been focused on doping III-V semiconductors into a DMS state. Although III-V DMS demonstrate the higher Curie temperature, $\mathrm{Mn}$ is much less soluble here than in II-VI semiconductors [1]. Another issue is that in III-V compounds magnetic doping harmfully affects emission properties and, moreover, changes conductivity to $p$-type. We have proposed a new approach to the problem based on using narrow band-gap III-V compounds possessing the largest intrinsic electronic $g$-factor, which are InSb and InAs [2]. Large electron $g$-factor, small effective electron mass and strong spin-orbit interaction at these semiconductors provide pronounced spin-related phenomena in absence of magnetic doping.

* corresponding author; e-mail: mihail-muhin@yandex.ru
Present research is focused on spin-related phenomena in low-dimensional $\mathrm{InSb} / \mathrm{InAs}$ heterostructures and continues our preliminary studies [3]. Magneto-optical investigations of type-II InSb quantum dots (QDs) in an InAs matrix and their theoretical consideration are reported.

\section{Experimental samples and technique}

$\mathrm{In}(\mathrm{Sb}, \mathrm{As})$ heterostructures incorporating monolayer-scale InSb insertions in an InAs matrix were fabricated by MBE. The InSb sub-monolayer insertions were formed by the InAs growth surface exposure to the $\mathrm{Sb}_{4}$ flux. Such process is expected to be possible due to efficient $\mathrm{As}-\mathrm{Sb}$ anion exchange reaction on the growth $\mathrm{InAs}$ surface. In the cross-section transmission electron microscopy (TEM) micrograph, the InSb sheets resemble ultrathin planar layers with faint thickness or composition inhomogeneities. The plan-view TEM image obtained for the structure with a single InSb sub-monolayer reveals an existence of quantum-dot-like InSb-rich islands. The islands characteristic lateral size and their sheet density are estimated as approximately $2.5 \mathrm{~nm}$ and $10^{12} \mathrm{~cm}^{-2}$, respectively. The details of the structures fabrication are described elsewhere [4].

Circularly polarized photoluminescence at external magnetic field applied in the Faraday geometry has been measured, allowing direct probing of the electron spin state. Experiments were carried out at magnetic field up to $5 \mathrm{~T}$ in the temperature range from 2 to $140 \mathrm{~K}$ and excitation density $0.1-40 \mathrm{~W} / \mathrm{cm}^{2}$. The samples were excited by a $809 \mathrm{~nm}$ line injection laser diode. The emission 
circular polarization was analyzed using a $\mathrm{NaCl}$ Fresnel rhomb followed with a linear polarizer. Photoluminescence (PL) spectra were detected by a grating monochromator with a nitrogen-cooled InSb photodiode.

\section{Experimental results}

Applying the external magnetic fields leads to splitting of PL contour from InSb QDs into two circular-polarized components and shifting toward higher energy. Increase of the magnetic fields brings about the polarization degree growth. At low magnetic fields $(B<2 \mathrm{~T})$ there is observed sublinear diamagnetic shift of the PL lines, at high magnetic fields increase of $B$ results in linear growth of the PL peak energy. Such behavior demonstrates well-known effect of magnetic freeze-out, which is typical for narrow gap semiconductors.

It was found that the polarization degree varies from $100 \% \sigma$-minus up to $10 \% \sigma$-plus, depending on the excitation intensity (Fig. 1) and temperature. At the lowest excitation intensity almost $100 \% \sigma$-minus polarization was detected. The polarization degree drops dramatically with the excitation intensity growth and changes its sign eventually. Actually, inversion point moves to lower intensities when sample temperature goes up. At high excitation intensity polarization degree saturates to a value of $10 \% \sigma$-plus.

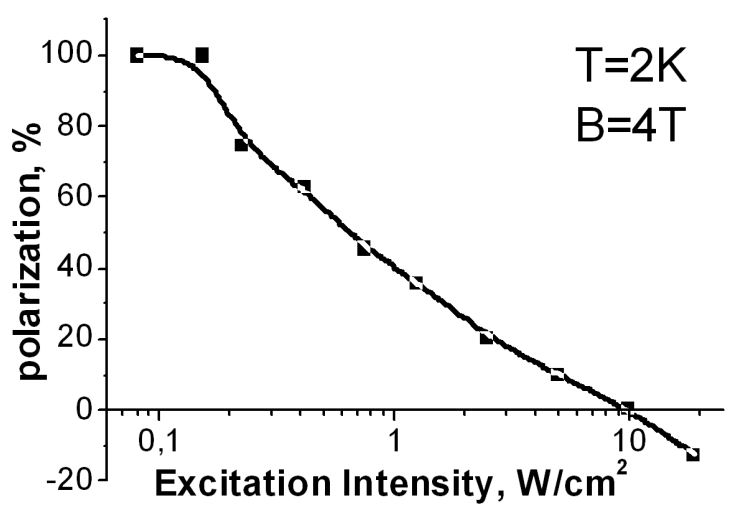

Fig. 1. Dependence of the polarization degree on excitation intensity at $T=2 \mathrm{~K}$ and $B=4 \mathrm{~T}$. The curve is a guide to the eye.

Temperature dependence of polarization degree is similar to excitation intensity dependence. Increase of the temperature leads to reduction of polarization degree and change of its sign. At the highest temperature polarization degree saturates to a value of $10 \% \sigma$-plus. A specific feature of temperature dependence is flat part of the curve at temperatures under $10 \mathrm{~K}$.

\section{Discussion}

The physical model of the phenomenon is based on the selection rules for optical transitions involving heavy holes, dependence of the oscillator strength on the excitation intensity, inherent for indirect in space optical transitions that only are allowed here, and an analysis of equilibrium population of energy levels. The detailed calculation of the energy spectrum of charge carriers, performed within a tight-binding approximation has been done. At this approach the InSb insert is considered as an ultrathin quantum well. The calculation shows that this system has a type II broken gap band alignment with holes localized on energy levels in quantum dots InSb and electrons located in the adjacent InAs coupled to holes via the Coulomb interaction. According to our calculation the ground state of InSb QDs is formed primarily by heavy holes with little admixture of light holes. So PL originates from recombination of the electrons in InAs and the heavy holes localized by InSb QDs.

Observed $100 \% \sigma$-minus PL polarization degree can be easily explained in terms of total spin alignment of electrons in InAs conduction band due to the Zeeman effect and selection rules for optical transition involving heavy holes in InSb. To explain reduction and final sign change of polarization degree if temperature is increased we have fitted experimental temperature dependence of polarization degree based on the analysis of equilibrium population of energy levels

$$
P=\frac{I^{\sigma-}-I^{\sigma+}}{I^{\sigma-}+I^{\sigma+}}=\tanh \left(\frac{\Delta E_{\mathrm{e}}+\Delta E_{\mathrm{hh}}}{2 k T}-\frac{\ln (f)}{2}\right),
$$

where $\Delta E_{\mathrm{e}}$ and $\Delta E_{\mathrm{hh}}$ - the Zeeman splitting of InAs conduction band electrons and the Zeeman splitting of heavy holes localized by InSb QDs, respectively, $f$ relative oscillator strength for optical transitions of electrons with different spin orientation.

The simulation of the experimental data revealed that the oscillator strength of the optical transitions of electrons with the spin oriented either along or against the magnetic field vector differs by approximately 1.8 times.

\section{Acknowledgments}

The financial support from Russian Ministry of Education and Science (No. 14.740.11.0463) is gratefully acknowledged.

\section{References}

[1] T.T. Chen, C.H. Chen, W.S. Su, M.H. Ya, Y.F. Chen, P.W. Liu, H.H. Lin, J. Appl. Phys. 93, 9655 (2002).

[2] Ya.V. Terent'ev, A.A. Toropov, B.Y. Meltser, A.N. Semenov, V.A. Solov'ev, I.V. Sedova, A.A. Usikova, S.V. Ivanov, Semiconductors 44, 194 (2010).

[3] Ya.V. Terent'ev, O.G. Lyublinskaya, A.A. Toropov, B.Ya. Meltser, A.N. Semenov, V.A. Solov'ev, S.V. Ivanov, Semiconductors 43, 635 (2009).

[4] S.V. Ivanov, V.A. Solov'ev, B.Ya. Meltser, Ya.V. Terent'ev, L.G. Prokopova, S.V. Ivanov, A.N. Semenov, J. Cryst. Growth 278, 203 (2005). 